\title{
Tag count based priority scheduling algorithm for mitigating the RFID collisions
}

\author{
Hema C, Dr.Sharmila Sankar, Sandhya M \\ Department of computer science and engineering, \\ B.S.Abdur Rahman Crescent Institute of Science and Technology, India
}

\begin{abstract}
Article Info
Article history:

Received Jan 10, 2019

Revised May 1, 2019

Accepted Jul 1, 2019

Keywords:

Cluster based RFID network Mobile reader collision tag count priority scheduling RFID

ABSTRACT

RFID (Radio Frequency IDentification) is a developing technology that employs electromagnetic signals to send the data among reader and tags to identify categorize and track the moveable items. The objective of this paper is to mitigate the reader collision problem by scheduling the readers in RFID system. Mobile readers in the RFID network send the same frequency signal to the air to read the data from the tags. While these two signals interfere each other and Tags are unable to backscatter signal to the reader. This causes a reader collision problem. The Reader collision problem reduces the lifetime of the RFID network and generates redundant data in the RFID Network. Tag Count based Priority Scheduling algorithm is proposed, that enhances the throughput of the readers and mitigates the reader collision problem. In the cluster based RFID network, The Dragonfly algorithm performed the Cluster Head reader election and cluster construction process and then allotting the mobile readers in the cluster. This algorithm improves the energy efficiency and diminishes the reader collision problem, thereby alleviating the tag information loss and expanding the mobile RFID network life time, while compared with Priority Clustering Protocol and the Graph Coloring based TDMA algorithm.
\end{abstract}

Copyright $@ 2020$ Institute of Advanced Engineering and Science. All rights reserved.

\section{Corresponding Author:}

Hema C,

Department of Computer Science and Technology,

B.S.Abdur Rahman Crescent Institute of Science and Technology,

Chennai, India.

Email: hemathanam@gmail.com

\section{INTRODUCTION}

RFID is a rising technology that includes the employs of electromagnetic spectrum to uniquely identify the objects, animals or humans. The objective of this paper is to mitigate the reader collision drawback by programming the readers in RFID system. RFID information contains plenty of duplication. The reader collision drawback happens once the signal from neighbor readers collides with the signal from other readers in the network. Such collisions may end up in lack of coordination among the readers and the tags. The Reader collision drawback will increase the energy and time consumption of RFID Reader thereby mitigate the lifespan of the RFID network and generates redundant information within the RFID Network that causes unneeded transmissions of redundant information. We diminishes the reader collision problem and enhances the energy efficiency of the mobile readers by proposing the new algorithm, Tag Count based Priority scheduling algorithm. Within the cluster based RFID network, The Cluster Head election and cluster formation method is completed by the dragonfly algorithm succeeded by organizing the mobile readers within the cluster through Tag Count Priority scheduling algorithm. Compared with the Graph Coloring based TDMA algorithm and Priority clustering Protocol (PCP), the proposed algorithm diminishes the reader collision and enhances the energy efficiency of the mobile readers, as a result mitigating the tag information loss and enhancing the life time of the RFID network. 
Radio frequency Identification is one among technologies that allow short vary, contact-less reading of information from a low price and compact data supply. An RFID system includes data-carrying transponders, referred to as tags and devices to access the information on the tags referred to as a reader or reader/writer. Tags are connected to the assets being monitored or carried by people. A reader is employed to receive information from the tags or to find the tag passing a specific location. Automatic Identification and Data Capture (AIDC) Technology is a method to identify and gather the information about a particular an item and use this information to carry out the various operations. Major reason is to perform all of this without human support. AIDC technologies are such as Voice Recognition, Barcodes, OCR, Smart cards, Magnetic Strips, Biometrics and RFID.

Whereas not the requirement of touching or seeing the information carrier, through the utilization of inductive coupling or magnetic force waves, The RFID is a technology, employed for gathering information about a particular item. The middleware software mainly used for reading and writing tags and therefore the tag are often improved by data encryption technique for security critical application and anti-collision algorithms are often enforced for the tags if many tags are to be read at the same time. The RFID systems are often divided into two key dimensions like technical infrastructure and logical infrastructure. The technical infrastructure includes the particular information capture technology comprised of tags, readers and transmission medium.

The logical infrastructure refers to the general identification method employed in representing objects. This logical infrastructure model relies on the principle of moving intelligence. The ID method includes the particular writing or naming system for objects and therefore the database contains the knowledge concerning the codes or identifications. RFID technology has several advantages over additional ancient approaches like faster read rates, not require physical line of sight, reads more than one tags at a time, Readable and Writable tags and improved security functionality.

RFID Tags are operate at three different types of radio frequency range such as Low Frequency range: $125 \mathrm{KHZ}$ or $134 \mathrm{KHZ}$, High Frequency range:13.56 MHZ and Ultra High Frequency range: 860-960 MHZ. This frequency ranges vary with country to country. The range of the low frequency wave is very low. The range up to $10 \mathrm{~cm}$. High Frequency wave can travel up to $1 \mathrm{~m}$. Ultra High Frequency radio waves can travel much longer and it ranges from $10 \mathrm{~m}$ to $15 \mathrm{~m}$.

The technical infrastructure of mobile RFID system accommodates of two components. One is a transceiver (transmitter/receiver) and antenna mixed as an mobile RFID reader and some other is a RFID tag consists of transponder (transmitter/responder) and antenna. Tags keep information about the item. Readers read the data from the tags.

A simple RFID system includes 3 components:

a) An antenna or coil used to convert electric powered electricity to radio waves.

b) A Reader used to store the data stored inside the tags by way of sending the sign to tags.

c) RFID tag used to store the information about the item.

There are essential aspects of RFID systems, one is reader collision and some other is tag collision is shown in Figure 1. Many usages need readers to function in closeness of every other reader. Due to the closeness, the signals from neighbor reader might collide with the signals from other readers in the mobile RFID network. This interference is referred to as reader collision. While a tag enters interference vicinity, reader electromagnetic fields overlap and tags are unable to reply reader queries. That is referred to as reader collision. The tag then turns into unresponsive and according to the type of tags and it cannot be detected until it leaves all readers fields.

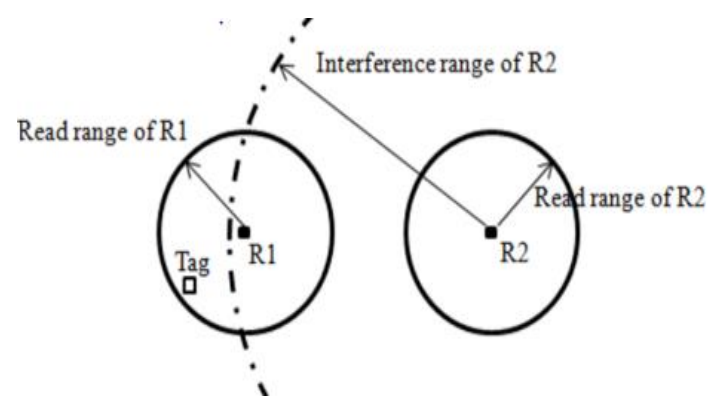

a) Reader to reader collision

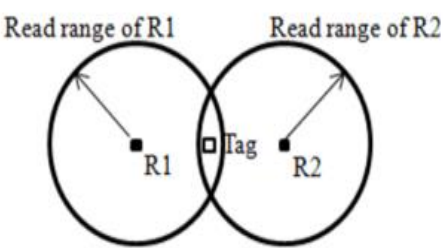

b) Reader to tag collision

Figure 1. Types of collision 
RFID readers transmit a sign to RFID tags. Tags acquire the readers signal and their replies can be correctly decoded by the reader is known as the interrogation area of the reader. The principle capability of an RFID reader is to find the specific identifiers of all the RFID tags in its interrogation sector. Simultaneous replies from RFID tags situated inside the interrogation zone of a reader make correct decoding of signals impossible. This problem, called the tag collision problem, prevents an RFID reader from concurrently reading all its protected RFID tags.

In existing approach, two cluster based scheduling protocols are used specifically Graph Coloring based TDMA algorithm and Priority clustering Protocol. In the Graph coloring algorithm, neighboring readers are not colored with identical color and also the same colored readers are read the data from the tags within the same slot, this evades the reader collision problem. However it enhances the energy and time efficiency of the mobile readers. In the priority clustering protocol, the priority is allotted to the each reader in the RFID network. Based on the priority of readers, they reads tags in sequence, it mitigates efficiency of the readers.

\section{RESEARCH METHOD}

Energy efficient cluster head selection method for information aggregation is employed for minimizing the reader mobility in RFID networks. In the cluster head selection method, all the readers within the network sent the energy state information to the server and it averages the energy and chosen the suitable cluster head readers. Optimum mobile cluster head readers are elected by applying the dragonfly algorithm to the suitable mobile cluster head readers. Remaining readers that don't seem to be chosen as a mobile cluster head reader, joined the cluster depends on the mobility of the cluster head reader. Subsequent to the cluster head selection and formation, the readers within the network are scheduled while not interfering from neighbor readers to read the data from the tag. To schedule the readers within the cluster based RFID network, we proposed new algorithm Tag count Priority scheduling algorithm.

\subsection{Dragonfly Clustering Protocol}

Energy efficient mobile cluster head reader selection theme for information aggregation ought to reduce the mobile reader mobility in RFID networks. Optimum mobile cluster head readers are chosen and unnecessary information is eradicated by information aggregation at the mobile cluster head reader. During this section, a brand new clustering approach referred to as "Dragonfly clustering approach" is introduced. The primary half during this describes the cluster head choice and cluster formation. The second half explains however reader scheduling is performed within the RFID network.

Dragonflies are the flowery insects. There are nearly 3000 totally different species of this type are found within the globe. Dragonflies are little predators, in nature it hunt several little insects in the world. Nymph dragonflies hunt different marine insects. This kind of species additionally hunts little fishes. This can be the distinctive and rare swarming behavior regarding dragonflies in comparison with different insects. Dragonflies swarm do two methods like looking the prey and migration from one swarm to a different. Dragonflies are static or dynamic in nature. Static swarm is thought as feeding swarm and dynamic is thought as migratory swarm. Dragonflies form a little cluster over a little space and flying front and back to hunt different flying preys like mosquitoes and butterflies. It produce sub-group at multiple areas to hunt the prey. The most aim of any swarm is survival in the world, thus all of the dragonflies move towards food sources and separated from enemies.

\subsection{Cluster Head Selection}

Each Reader and tag contains potential score like similarity of mobility and residual energy. Residual energy of the reader is that the remaining energy of the reader within the network. The reader with high residual energy is chosen as a cluster head reader. Mobility is additionally taken under consideration for reducing frequent cluster breakage before information transmission. Mobile RFID reader Cluster construction will elongate the life span of the RFID network. Mobile cluster head readers perform information aggregation before information sent to the server. An arbitrarily deployed device network needs a cluster formation protocol to partition the RFID network into clusters. The mobile cluster head readers are chosen within the clusters so as to evade the energy consumption from all mobile readers. There are two approaches utilized in this method, the cluster head reader initial and also the cluster initial approach. In the cluster head reader initial approach, the cluster head is chosen initial so cluster is created. In the cluster initial approach the cluster is created initial and then the mobile cluster head reader is chosen.

The cluster head readers are chosen based on the potential score like energy, speed and direction. The mobile reader that has high energy is chosen as a mobile cluster head reader. Mobility is another necessary factor for choosing the cluster head reader within the cluster. If the reader has high totally different 
mobility with high energy, then it cannot be chosen as cluster head. All the readers can send the energy data to the base station. Based on the typical energy, eligible readers are chosen. Once applying the dragonfly algorithm, the optimum readers are chosen as a cluster head.

\subsection{Optimum Mobile Cluster Head Reader Selection}

Separation, Alignment and Cohesion values are calculated in dragonfly algorithm to optimum mobile cluster head election, as soon as eligible mobile cluster head election as shown in the Figure 2 Separation formula is used to calculate the distance between the eligible mobile cluster head reader and member readers. Separation formula is utilized to mitigate reader collision between the mobile readers within the RFID network. Reader collision is avoided by maintaining the distance between the readers within the network. The separation equation is mentioned below

$$
\mathrm{S}_{\mathrm{i}}=\left(\sqrt{(X 2-X 1)^{2}+(Y 2-Y 1)^{2}}\right)
$$

In the above separation equation, (X1, Y1) represents the location of the particular mobile reader. (X2, Y2) represents the location of the neighboring mobile readers in the RFID network. In the cluster, mobility of cluster head mobile reader and neighbouring mobile readers are calculated to check if they are in the same direction. The cluster breakage problem happens in the cluster, when the mobility of the cluster head mobile reader is totally different from the neighboring mobile readers in the network. The mobility of mobile reader is maintained within RFID network, to avoid the cluster breakage problem in the network. The alignment is computed by the equation given

$$
\mathrm{A}_{\mathrm{i}}=\left(\sum_{j=1}^{N} \mathrm{Vj}_{j}\right) / \mathrm{N}
$$

In the above Alignment equation, $\mathrm{Vj}$ represents the mobility of $\mathrm{j}$-th neighboring mobile reader in the RFID network. $\mathrm{N}$ shows the number of neighbor reader among the readers. Neighbor count is calculated for the eligible cluster in cohesion factor. The cohesion equation is mentioned below:

$$
\mathbf{C}_{\mathrm{i}}=\left(\sum_{\mathrm{j}=1}^{\mathrm{W}} \mathrm{Xj}\right) / \mathbf{N}-\mathbf{X}
$$

In the above equation $\mathrm{X}$ represents the location of the current individual, $\mathrm{N}$ represents the count of neighboring readers and $\mathrm{Xj}$ shows the location of $\mathrm{j}$-th neighboring reader in the mobile RFID network. Optimum mobile cluster head reader is selected from the eligible mobile cluster head readers based on the separation, alignment and cohesion values.
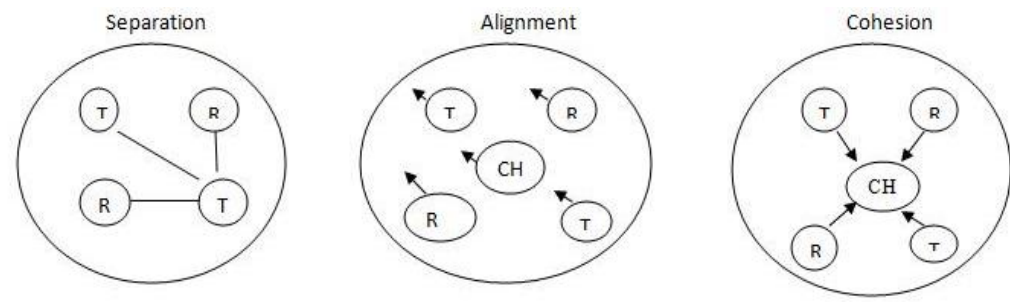

Figure 2. Techniques used in dragonfly algorithm

\subsection{Joining the Cluster Head}

The cluster head reader can prolong the life time of the mobile readers and the RFID network. Once the cluster heads are chosen, they transmit the message to all readers and tags. The remaining readers those are not elected as cluster head readers will form the cluster depend on the mobility. Once the cluster formation is finished, Tag information transmission starts inside the cluster. Once readers read the data from tag, the readers will transmit the data to the cluster head readers.

\subsection{Scheduling the Readers}

Subsequently the cluster head election and construction, the member mobile RFID readers in the network are scheduled without interfering from neighboring readers to read the information from the tag. To schedule the readers in the cluster based RFID network, we proposed new algorithm Tag count Priority 
scheduling algorithm. In the cluster, each RFID member reader sends their Tag count to the cluster head reader. Depend on the tag count of the neighbor readers, mobile RFID cluster head reader assigns the priority to the each member reader in the cluster. It assigns high priority to reader which has maximum Tag count and assigns low priority to the reader which has minimum tag count.

In the Tag count Priority scheduling algorithm, High priority reader reads the information from tags in the time slot1. At the same time, the member readers those are not in interfere with high priority reader also reads the information from the tags in the same time slot. Then the Next High priority reader repeats the steps in the time slot2. This process is repeated until all the readers read the information from tags in the cluster.

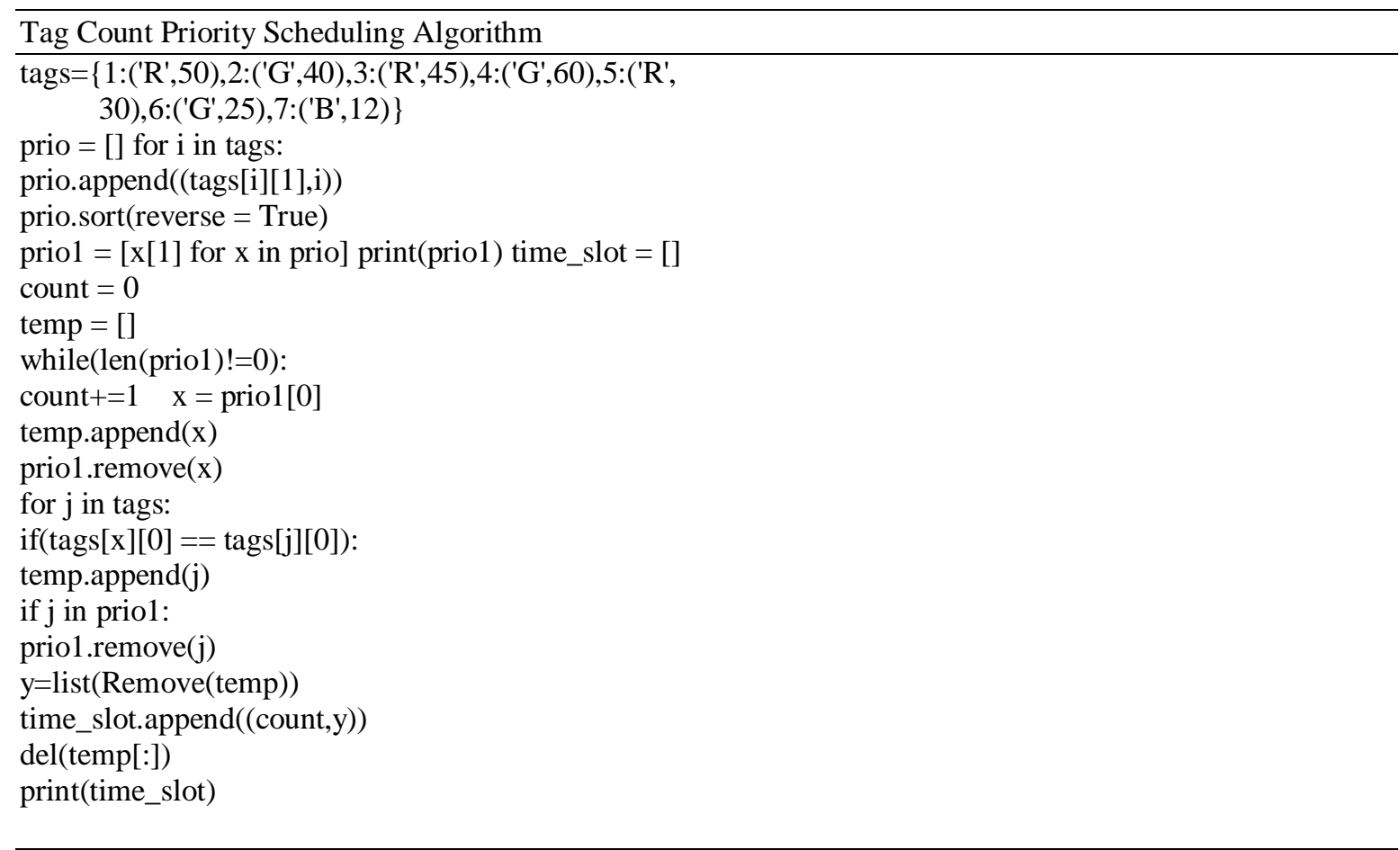

In the Figure 3, the cluster consists of six RFID readers such as R1, R2, R3, R4, R5 and R6. Each member reader sends their tag count to the cluster head reader. Tag count of the reader is defined as number of tags around the reader. Based on the tag count, cluster head reader assigns high priority to the reader $\mathrm{R} 1$; low priority is assigned to reader $\mathrm{R} 6$.

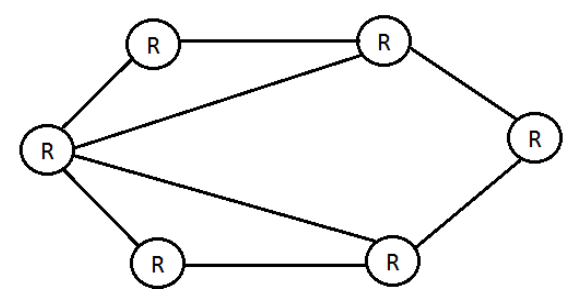

Figure 3. Cluster based RFID Network

RFID reader contains number 6 , which represents high priority The number of the member readers in the cluster is equal to 6 so cluster head reader assign number 6 for high priority, number 1 for low priority, number 5 for next high priority and number 2 for next low priority and so on as shown in the Table 1 .

In the Scheduling table, high priority reader reads the information from the tags in the time slot 1. Refer the cluster based RFID network diagram in Figure 5. There is an edge between reader R1 and R2, which represents there is interference between reader R1 and R2. The readers R3, R4 and R5 are also in the interference range of $\mathrm{R} 1$. The reader $\mathrm{R} 6$ is not in the interference range of reader $\mathrm{R} 1$ hence it reads the information from tags while Reader R1 reads during time slot 1 as shown in Table 2. 


\begin{tabular}{ccccccc}
\multicolumn{1}{c}{ Table } & 1 . Priority Table \\
\hline Priority & 6 & 5 & 4 & 3 & 2 & 1 \\
Tag count & 55 & 40 & 33 & 22 & 12 & 6 \\
\hline
\end{tabular}

Table 2. Scheduling Table

\begin{tabular}{ccc}
\hline Time slot1 & Time slot2 & Time slot3 \\
\hline R1 & R2 & R4 \\
R6 & R3, R5 & - \\
\hline
\end{tabular}

At the time slot 2, the next high priority Reader R2 is in interference with reader R1 and R4. Since Reader R1 has already read the information, the readers R3 and R5 reads the information from tags. Finally Reader R4 reads the information from tags in the time slot 3.

\section{SIMULATION RESULTS}

Throughput, RFID Tag Delivery rate, end-to-end delay, and energy consumption factors are utilized to evaluate the effectiveness of Tag count based priority scheduling algorithm and it is compared with existing scheduling algorithm such as Priority Clustering Protocol (PCP) and Graph coloring based TDMA algorithm.

\subsection{Throughput}

Throughput refers to how much information can be read from the tag in a given amount of time. It is used to measure the performance of the Tag Count Priority Scheduling algorithm and Throughput of the proposed algorithm usually measured in bits per second. The Table 3. shows that Tag Count based scheduling algorithm has better throughput, compared with Priority Clustering Protocol (PCP) and Graph coloring based TDMA algorithm.

Figure 4 shows the graph based on the Throughput Comparison. In the Comparative graph, $\mathrm{x}$ axis indicates the Throughput in kbps and the y axis indicates Time in milliseconds. It displays that the Tag count based priority scheduling algorithm has better throughput When compared to Priority Clustering Protocol (PCP) and Graph coloring based TDMA algorithm.

Table 3. Throughput Comparison

\begin{tabular}{cccc}
\hline Time $(\mathrm{ms})$ & TCPS & GC_TDMA & PCP \\
\hline 10 & 0.035 & 0.030 & 0.025 \\
20 & 0.038 & 0.035 & 0.030 \\
30 & 0.040 & 0.038 & 0.035 \\
40 & 0.050 & 0.045 & 0.040 \\
50 & 0.055 & 0.045 & 0.040 \\
60 & 0.051 & 0.045 & 0.040 \\
70 & 0.054 & 0.048 & 0.040 \\
80 & 0.059 & 0.052 & 0.042 \\
90 & 0.061 & 0.056 & 0.046 \\
\hline
\end{tabular}

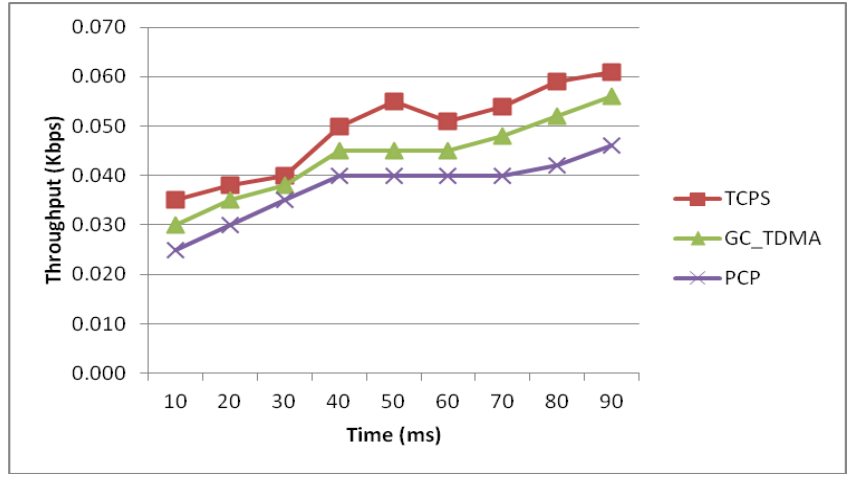

Figure 4. Throughput comparative graph

\subsection{End to end Delay}

The performance improvement is measured with respect to the end to end delay as shown in Table 4. and Figure 5. represents End to end delay comparative graph. Below mentioned graph, $\mathrm{x}$ axis shows time in milliseconds and y axis shows delay rate in kbps. The average time taken by reader to read the information from tag and successfully delivered to the destination. It also includes the delay caused by scheduling the readers in the cluster process and Tag information transmission. Only the Tag information that successfully transmitted to destinations that calculated.

Since the mobile RFID readers could discover the difficult to interrogate the information from the tags and so increasing the delay, we elected mobile RFID cluster head reader and constructed cluster. It is simple for the mobile readers to interrogate the data from tags with the smallest delay by scheduling and allotting the mobile readers in the time slot. 


\begin{tabular}{ccc}
\multicolumn{3}{c}{ Table 4. End to end Delay Comparison } \\
\hline Time (ms) & GC_TDMA & PCP \\
\hline 10 & 0.012 & 0.014 \\
15 & 0.025 & 0.020 \\
20 & 0.025 & 0.010 \\
25 & 0.022 & 0.012 \\
30 & 0.019 & 0.019 \\
35 & 0.018 & 0.017 \\
40 & 0.018 & 0.021 \\
50 & 0.018 & 0.024 \\
60 & 0.018 & 0.025 \\
70 & 0.018 & 0.027 \\
80 & 0.018 & 0.030 \\
\hline
\end{tabular}

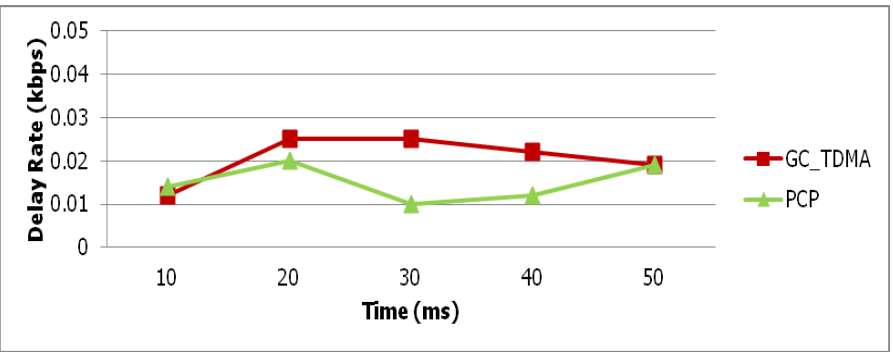

Figure 5. End to end delay comparative graph

\subsection{RFID Tag delivery rate}

The rate of number of tag information that is effectively transmitted to the cluster head reader with number of tag information that has been read by the reader. The reader collision is minimized by the packet delivery rate. The reader collision is avoided by the cluster formation by Dragonfly algorithm succeeded by organizing the member readers in the cluster by Tag Count Priority Scheduling algorithm, thereby reducing the energy and time consumption of the readers in the RFID network. While executing the Priority Clustering Protocol and Graph Coloring based TDMA algorithm, extra time and energy is consumed on classifying and allocating the time slot for the mobile RFID readers. End to end delay comparison as shown in Table 5.

Figure 6 represents the comparative graph of the packet delivery ratio. The results represent that the packet delivery ratio of the readers using the Tag Count based Priority Scheduling algorithm is very high when compare to the Graph coloring based TDMA algorithm and Priority Clustering Protocol. Tag Count based Priority Scheduling algorithm shows considerable performance improvement. Reader collision problems generally appear in the dense mobile reader environment, when more than one mobile readers attempt to interrogate tags concurrently in the equal area. The read results can be unacceptable with read times and a utilization of energy.

For the purpose of defeat this problem and to maintain the utilization of energy, mobile readers are arranged as clusters and The mobile RFID Cluster Head reader election and cluster construction process is performed with the Dragonfly algorithm succeeded by arranging the member readers in the cluster using Tag Count Priority Scheduling algorithm. Both Graph coloring based TDMA and Priority clustering Protocol increases the energy and time consumption while compared to Tag count based Priority scheduling algorithm.

Table 5. End to end delay Comparison

\begin{tabular}{cccc}
\hline Time $(\mathrm{ms})$ & TCPS & GC_TDMA & PCP \\
\hline 10 & 36 & 30 & 22 \\
20 & 48 & 40 & 30 \\
30 & 65 & 60 & 50 \\
40 & 82 & 78 & 70 \\
50 & 86 & 80 & 75 \\
60 & 89 & 82 & 80 \\
70 & 91 & 85 & 81 \\
80 & 96 & 92 & 81 \\
90 & 96 & 92 & 81 \\
100 & 98 & 92 & 81 \\
\hline
\end{tabular}

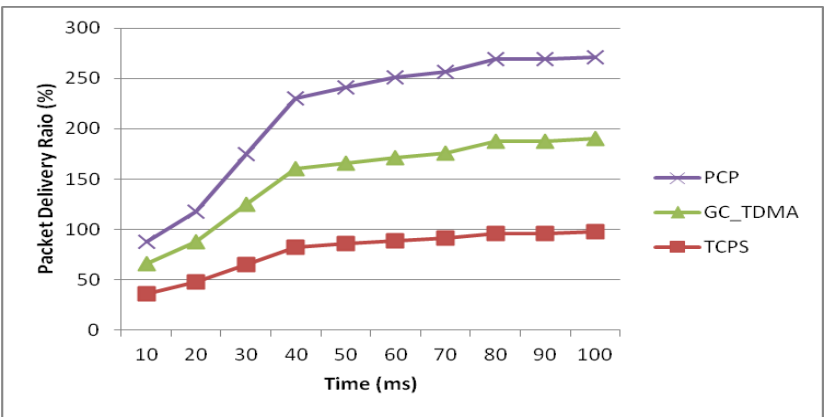

Figure 6. RFID tag delivery rate comparative graph

\section{CONCLUSION}

The efficiency of the reader and stability of the reader is improved by cluster head selection and cluster formation scheme using Dragon Fly algorithm. To avoid the reader collision and reduce the network traffic by scheduling the readers in the cluster based RFID network, we proposed Tag Count Priority Scheduling algorithm. The scheduling algorithm used in this paper helps to reduce the reader collision problem, thereby reducing energy and time consumption of the readers. The performance of Tag Count Priority Scheduling algorithm has been measured and reported with reference to different parameters such as Packet delivery ratio, Throughput and Average end to end delay. Results indicated that our Tag Count Priority Scheduling performs better when compared with reader anti-collision scheduling algorithms such as 
Graph Coloring based TDMA scheduling algorithm and Priority Clustering Protocol. In the future, we will form clusters in the Heterogeneous RFID networks and reduces the reader collision problems in the cluster based RFID network by increasing the Throughput and Packet delivery ratio.

\section{REFERENCES}

[1] H.A.Ahmed, H.Salah and J.Robert, “ Closed-Form Solution For Aloha Frame Length Optimizing Multiple Collision Recovery Coefficients' Reading Efficiency”, IEEE Systems Journal, Vol. 12, No.1, pp. 1047-1050, 2018.

[2] Xi Tan, He Wang and Lingzhi Fu, "Collision detection and signal recovery for UHF RFID systems", IEEE Transactions on Automation Science and Engineering, Vol.15, No. 1, pp. 239-250, 2018.

[3] Jian Su, Zhengguo Sheng and Liangbo Xie,"AA collision-tolerant based anti-collision algorithm for large scale RFID system”, IEEE Communication Letters, Vol. 21, No. 7, pp. 1517-1520, 2017.

[4] Mehdi Golsorkhtabaramiri and Neda Issazadeh Kojid,"A Distance Based RFID Reader Collision Avoidance Protocol for Dense Reader Environments”, Wireless Pers Comm Springer, Vol. 95, No. 2, pp. 1781-1798, 2017.

[5] Atef Jaballah and Aref Meddeb, "Algorithm for readers arrangement without collision in RFID networks", International Conference on Parallel and Distributed Computing, Applications and Technologies, pp. 316-321, 2017.

[6] $\mathrm{He} \mathrm{Xu}$, Weiwei Shen and Peng Li, "A novel algorithm L-NCD for redundant reader elimination in P2P-RFID network", Journal Of Algorithms \& Computational Technology, Vol. 11, No. 2, pp. 135-147, 2017.

[7] He Xu, Weiwei Shen, Peng Li, Jiawei Zhu, and Ruchuan Wang," A novel algorithm 1-ncd for redundant reader elimination in p2p-rfid network", Journal of Algorithms \& Computational Technology", Vol.11, pp.135-147, 2017.

[8] Ngoc-Tu Nguyen, Bing-Hong Liu, And Van-Trung Pham, "A Dynamic-Range-Based Algorithm for Reader-Tag Collision Avoidance Deployment in RFID Networks", International Conference on Electronics, Information, and Communications (ICEIC), 2016.

[9] Bing Hong Liu, Ngoc Tu Nguyen and Van Trung Pham Yeh, "A Maximum Weight Independent Set Based Algorithm for Reader-Coverage Collision Avoidance Arrangement in RFID Networks", IEEE Sensors Journal, Vol. 16, No. 5, pp. 1342-1350, 2016.

[10] Yi Jiang, Ruonan Zhang, Wei Cheng and Wei Sun, "An Efficient Multi-Channel Reader Collision Avoidance Protocol in RFID Systems", IEEE Wireless Communications and Networking conference, 2016.

[11] J.Vales Alonso, F.J.Parrado Garca and J.J.Alcaraz, "OSL:An optimization-based scheduler for RFID dense-reader environments", Ad Hoc Networks, Vol. 37, pp. 512-525, 2016.

[12] Jian Su, Zhengguo Sheng and Danfeng Hong, "An effective frame breaking policy for dynamic framed slotted Aloha in RFID”, IEEE Communication Letters, vol. 20, no. 4, p. 692-695, 2016.

[13] Haidarsafa, Wassim El-Hajj and Christine Meguerditchian, "A distributed multi-channel reader anti-collision algorithm for RFID Environments", ELSEVIER, 2015.

[14] Zhonghua Li, Hanpei Yang, Jianming Li, Chunhui He and Jieying Zhou, “ An Enhanced Neighbour-friendly Reader Anti-collision Algorithm in Mobile RFID Networks”, IEEE, pp. 238-241, 2014.

[15] Ahmed Jedda and Hussein T. Mouftah, "Decentralized RFID Coverage Algorithms with Applications for the Reader Collisions Avoidance Problem”, IEEE Transactions on Emerging Topics in Computing, 2015.

[16] P.Varalakshmil, R.Nandakumar and M.Umadevi, "An Efficient Cluster Head Selection and Aggregation for Wireless Sensor Networks", IEEE International Conference on Communication and Signal Processing, 2014.

[17] Faiza Nawaz, Varun Jeoti, Azlanawang and Michealdrieberg, "Reader to Reader Anti-collision Protocols in Dense and Passive RFID Environment", IEEE 11th Malaysia International Conference on Communications, pp. 468-473, 2013.

[18] Juan J. Alcaraz, Javier Vales-Alonso and Joan Garcia-Haro, "RFID Reader Scheduling for Reliable Identification of Moving Tags", IEEE Transactions on Automation Science and Engineering, Vol. 10, No. 3, pp. 816-828, 2013.

[19] Sinemcoleriergen, Pravin Varaiya,"TDMA scheduling algorithms for wireless sensor networks", Springer, pp. 985-997, 27 May 2009.

[20] Shailesh M. Birari and Sridhar Iyer, "Mitigating the Reader Collision Problem in RFID Networks with Mobile Readers", IEEE, pp. 463-468, 2005.

[21] Hyunsook and Kim, "An Efficient Clustering Scheme for Data Aggregation Considering Mobility in Mobile Wireless Sensor Networks", International Journal of Control and Automation, Vol. 6, No.1, pp. 1-14, February 2013.

[22] Vivek Chandran and Nikesh, "Elimination of Data Redundancy and Latency Improving in Wireless Sensor Networks", International Journal of Engineering Research and Technology, Vol. 3, No.6, pp. 8-15, August 2014.

[23] Mohamed Watfa, William Daher and Hsham Al Azar, "A Sensor Network Data Aggregation Technique", International Journal of Computer Theory and Engineering, Vol. 1, No. 1, pp. 67-80, April 2013.

[24] Bala Krishna and Noble Vashishta, "Energy Efficient Data Aggregation Techniques in Wireless Sensor Networks", 5th International Conference on Computational Intelligence and Communication Networks, Vol. 5, No. 1, pp. 23-28, March 2013.

[25] Faiza Nawaz, Varun Jeoti, Azlan Awang and Micheal Drieberg, "Reader to Reader Anti collision Protocols in Dense and Passive RFID Environment", IEEE 11th Malaysia International Conference on Communications, Vol. 2, No. 5, pp. 754-771, 2013.

[26] Zhonghua Li, Hanpei Yang, Jianming Li, Chunhui He and Jieying Zhou, “ An Enhanced Neighbor-friendly Reader Anti-collision Algorithm in Mobile RFID Networks”, IEEE, pp. 238-241, 2014. 
[27] Juan J. Alcaraz, Javier Vales-Alonso and Joan Garcia-Haro, "RFID Reader Scheduling for Reliable Identification of Moving Tags", IEEE Transactions on Automation Science and Engineering, Vol. 10, No. 3, pp. 816-828, July 2013.

[28] Waleed Alsalih, "Discrete Power-Based Distance Clustering for Anti-Collision Schemes in RFID Systems", 13th Annual IEEE Workshop on Wireless Local Networks, pp. 868-873, 2013.

[29] Ji Peng, Li Yupeng, Jiang Jingqi and Wang Tianbao, "A Clustering Protocol for Data Aggregation in Wireless Sensor Network", International Conference on Control Engineering and Communication Technology, Vol. 54, No. 1, pp. 978-991,2012.

[30] Meenakowshalya and Sukanya, "Clustering Algorithms for Heterogeneous Wireless Sensor Networks", Journal of Ad Hoc, Sensor and Ubiquitous Computing, Vol. 2, No. 3, pp. 570-576, 2011.

[31] Shweta Jain and Samir R. Das, "Collision Avoidance in a Dense RFID Network", The 7th International Conference on Networking, Vol. 3, No. 6, pp. 28-40, 2010.

[32] Nazish Irfan,Mustapha and Yagoub, "Efficient Algorithm for Redundant Reader Elimination in Wireless RFID Networks”, International Journal of Computer Science Issues, Vol. 7, No. 11, pp. 4-16, 2010.

[33] Shaojie Tang, Cheng Wang, Xiang-Yang Li, and Changjun Jiang, "Reader Activation Scheduling in Multi-Reader RFID Systems: A Study of General Case", IEEE International Parallel \& Distributed Processing Symposium, pp. 1147-1155, 2011

[34] J. Vales-Alonso, M. V. Bueno-Delgado and J. J. Alcaraz, "Optimal scheduling in dual reader RFID Environments", IEEE International Conference on RFID-Technologies and Applications, pp. 479-485, 2011.

[35] Chun-Fu Lin and Frank Yeong-Sung Lin, "Efficient Estimation and Collision-Group-Based Anticollision Algorithms for Dynamic Frame-Slotted ALOHA in RFID Networks", IEEE Transactions on Automation Science and Engineering, Vol. 7, No. 4, pp. 840-848, 2010.

[36] Jun-Bong Eom, Soon-Bin Yim, and Tae-Jin Lee, "An Efficient Reader Anticollision Algorithm in Dense RFID Networks With Mobile RFID Readers", IEEE Transactions on Industrial Electronics, Vol. 56, No. 7, pp. 2326-2336, 2009.

[37] SinemColeriErgen and Pravin Varaiya, "TDMA scheduling algorithms for wireless sensor networks", Springer, pp. 985-997, 2009.

[38] Younis and Fahmy, "HEED: Hybrid, Energy-Efficient, Distributed Clustering Approach for Ad hoc Sensor Networks", IEEE Transactions on Mobile Computing, Vol. 5, No. 8, pp. 366-379, 2002.

[39] W. R. Heinzelman, A. Chandrakasan and H. Balakrishnan, "Energy Efficient Communication Protocol for Wireless Microsensor Networks", The 3rd Annual Hawaii International Conference on System Sciences, Vol.13, No. 6, pp. 36-43, 2000 .

\section{BIOGRAPHIES OF AUTHORS}

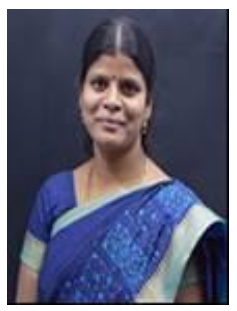

Hema C received the Master degree in computer science \& engineering from Anna University in 2007. She is a research student of B.S.Abdur Rahman Crescent Institute of Science and Technology. Currently, she is an Assistant Professor (Sr.G) at B.S.Abdur Rahman Crescent Institute of Science and Technology. Her research interests are RFID, Wireless sensor networks.

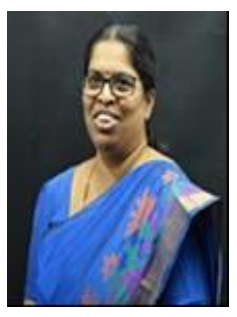

Dr. Sharmila Sankar received the degree in computer science \& engineering from Madras University in 2002. She received the Ph.D. degree in computer science \& engineering from the Anna University in 2012. Currently, she is the Professor at B.S.Abdur Rahman Crescent Institute of Science and Technology. Her research interests include Adhoc networks, RFID, wireless sensor networks.

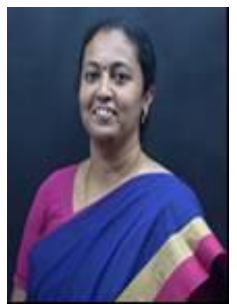

Dr. Sandhya M received the degree in computer science \& engineering from Madras University in 2002. She received the Ph.D. degree in computer science \& engineering from the Anna University in 2012. Currently, she is the Head and Professor at B.S.Abdur Rahman Crescent Institute of Science and Technology. Her research interests include Adhoc networks, RFID, wireless sensor networks 